\title{
Evidence of a cellulosic layer in Pandoravirus tegument and the mystery of the genetic support of its biosynthesis
}

Running title: the cellulosic tegument of Pandoravirus

2 Author list: Djamal BRAHIM BELHAOUARI ${ }^{1}$, Jean-Pierre BAUDOIN ${ }^{1}$, Franck

3 GNANKOU ${ }^{1}$, Fabrizio DI PINTO ${ }^{1}$, Philippe COLSON ${ }^{1,2}, \operatorname{Sarah}_{\text {AHERFI }}{ }^{1,2} *$, Bernard LA

$4 \operatorname{SCOLA}^{1,2 *}$

$5{ }^{1}$ Aix-Marseille Univ., Institut de Recherche pour le Développement (IRD), Assistance Publique -

6 Hôpitaux de Marseille (AP-HM), MEPHI, 27 boulevard Jean Moulin, 13005 Marseille, France

$7 \quad{ }^{2}$ IHU Méditerranée Infection, 19-21 boulevard Jean Moulin, 13005 Marseille, France

9 * Correspondence: $\underline{\text { sarah.aherfi@ap-hm.fr and bernard.la-scola@ univ-amu.fr }}$ 


\section{Abstract}

12 Pandoraviruses are giant viruses of amoebae with $1 \mu \mathrm{m}$-long virions. They have an ovoid

13 morphology and are surrounded by a tegument-like structure lacking any capsid protein nor any gene

14 encoding a capsid protein. In this work, we studied the ultrastructure of the tegument surrounding

15 Pandoravirus massiliensis virions and noticed that this tegument is composed of a peripheral sugar

16 layer, an electron-dense membrane, and a thick electron-dense layer consisting in several tubules

17 arranged in a helicoidal structure resembling that of cellulose. Pandoravirus massiliensis particles

18 were stained by Calcofluor white, a fluorescent dye of cellulose, and the enzymatic treatment of

19 particles by cellulase showed the degradation of the viral tegument. We first hypothesized that the

20 cellulose tegument could be synthesized by enzymes encoded by Pandoravirus. Bioinformatic

21 analyses revealed in Pandoravirus massiliensis, a candidate gene encoding a putative cellulose

22 synthase, with a homology with the BcsA domain, one of the catalytic subunits of the bacterial

23 cellulose synthase, but with a low level of homology. This gene was transcribed during the

24 replicative cycle of Pandoravirus massiliensis, but several arguments run counter to this hypothesis.

25 Indeed, even if this gene is present in other Pandoraviruses, the one of the strain studied is the only

26 one to have this BcsA domain and no other enzymes involved in the synthesis of cellulose could be

27 detected, although we cannot rule out that such genes could have been undetected among the large

28 proportion of Orfans of Pandoraviruses. As an alternative, we investigated whether Pandoravirus

29 could divert the cellulose synthesis machinery of the amoeba to its own account. Indeed, contrary to

30 what is observed in the case of infections with other giant viruses such as mimivirus, it appears that

31 the transcription of the amoeba, at least for the cellulose synthase gene, continues throughout the

32 growth phase of envelopes of Pandoravirus. Finally, we believe that this scenario is more plausible. If

33 confirmed, it could be a unique mechanism in the virosphere.

34 Key words: Giant virus, Pandoravirus, amoeba, Acanthamoeba, Megavirales, capsid, tegument 
cellulose.

\section{Introduction}

Giant viruses of amoebae are a group of complex viruses phylogenetically closely relative to the Nucleo - Cytoplasmic Large DNA Viruses (NCLDVs), within a new proposed order named

39 Megavirales (Colson et al., 2013). Since the discovery of the first giant virus of amoebae in 2003 (La

40 Scola et al., 2003), dozens of new giant viruses were described, considerably expanding our

41 knowledge about their diversity, structure, genomics and evolution.

In 2013, two new complex giant viruses, named pandoraviruses, were described (Philippe et

al., 2013). They replicate in Acanthamoeba castellanii and compose a new phylogenetic group of

giant viruses of amoebae related to phycodnaviruses. The first isolate, named Pandoravirus salinus,

originated from a marine sediment layer of the Tunquen River in Chile. The second isolate,

Pandoravirus dulcis, was isolated from the mud of a freshwater pond in Australia. Pandoraviruses

harbor specific morphological and genetic features, including ovoid-shaped particles with an ostiole-

like apex and measuring $\sim 1.0 \mu \mathrm{m}$ in length and $\sim 0.5 \mu \mathrm{m}$ in diameter, classifying them as the second

largest particles after those of Pithovirus sibericum (Legendre et al., 2014). These viruses have a

double-stranded DNA genome, up to $2.5 \mathrm{Mb}$ (for Pandoravirus salinus), the largest genome ever

51 described to date in the virosphere (Philippe et al., 2013). Subsequently, the nature of an

52 endocytobiont of Acanthamoeba isolated few years before in Germany from the contact lens and

storage case fluid of a patient with keratitis, was recognized as the third pandoravirus and was named

54 Pandoravirus inopinatum (Scheid, 2016; Scheid et al., 2014). In 2015-2016, we isolated three new

pandoravirus strains from sewage and soda lake water samples collected in Brazil, by co-culture on

A. castellanii. These viruses were named respectively Pandoravirus massiliensis, Pandoravirus

pampulha, and Pandoravirus braziliensis (Aherfi et al., 2018). Other recent prospecting studies 
collected in Marseille (France); Pandoravirus neocaledonia, isolated from the brackish water of a

mangrove near Noumea airport (New Caledonia), Pandoravirus macleodensis, isolated from a

61 freshwater pond near Melbourne (Australia) (Legendre et al., 2018), Pandoravirus celtis, isolated

62 from a soil sample collected in Marseille (Legendre et al., 2019) and 3 new pandoraviruses isolated

63 from water samples in Brazil (Pereira Andrade et al., 2019).

64 Although other giant viruses with a similar ovoid morphology have been described, including

65 pithoviruses, cedratviruses or Orpheovirus (Andreani et al., 2016, 2018; Legendre et al., 2014),

66 pandoraviruses have the intriguing particularity to harbor no hints of a known capsid protein and, at

67 the same time, the absence in virions of any structure similar to that of a known capsid (Aherfi et al.,

68 2018; Legendre et al., 2018; Philippe et al., 2013; Scheid, 2016). In addition, no capsid-resembling

69 protein was identified by proteomics in the Pandoravirus salinus and Pandoravirus massiliensis

70 virions (Aherfi et al., 2018; Philippe et al., 2013). Pandoravirus virions are wrapped in a $\approx 70$-nm-

71 thick tegument-like envelope, composed of three layers: one $\approx 20 \mathrm{~nm}$-thick of light density; one

72 intermediate of $\approx 25 \mathrm{~nm}$-thick that appears darker and composed of fibrils; and an external layer $\approx 25$

73 nm-thick with a medium density (Philippe et al., 2013). At one of the particle apex, an aperture of

$74 \approx 70 \mathrm{~nm}$ in diameter opens the viral tegument. An internal lipid membrane is present beneath the

75 tegument, thus delimiting the particle core. The tegument and the internal content of pandoravirus

76 virions are synthesized simultaneously, from the aperture-harboring apex, during neo-virion synthesis

77 in the cytoplasm of the amoebae. However, the nature of this tegument has remained unknown to

78 date. Its deciphering is needed to refine the definition of these giant viruses.

79 In the present study, we aimed to characterize the nature of the tegument of pandoraviruses.

\section{Material and methods}


82 For negative staining, a drop of purified Pandoravirus massiliensis particles fixed with 2.5\%

83 glutaraldehyde in $0.1 \mathrm{M}$ cacodylate buffer was adsorbed onto formvar carbon films on 400 mesh

84 nickel grids (FCF400-Ni, EMS). Grids were stained for 10 seconds with $1 \%$ molybdate solution in

85 filtered water at room temperature. For sections, pandoravirus particles were ultracentrifugated at

$868,000 \mathrm{~g}$ for 10 minutes and fixed for $1 \mathrm{~h}$ at $4{ }^{\circ} \mathrm{C}$ under gentle mixing after pellet resuspension in a

87 mixture of $1.2 \%$ glutaraldehyde / $0.05 \%$ ruthenium red in $0.1 \mathrm{M}$ cacodylate buffer. Virus particles

88 were ultracentrifugated at $8,000 \mathrm{~g}$ for 10 minutes to discard supernatant and were then fixed for $3 \mathrm{~h}$ at

$894{ }^{\circ} \mathrm{C}$ in a mixture of $1.2 \%$ glutaraldehyde $/ 0.05 \%$ ruthenium red in $0.1 \mathrm{M}$ cacodylate buffer. Then,

90 Pandoravirus massiliensis virions were washed thrice $10 \mathrm{~min}$ with $0.1 \mathrm{M}$ Cacodylate buffer at $4{ }^{\circ} \mathrm{C}$.

91 Next steps were performed at room temperature. Viral particles were rinsed twice for 15 min each,

92 with a cacodylate $0.1 \mathrm{M} /$ saccharose $0.2 \mathrm{M}$ in water solution, and were dehydrated with ethanol

$9350 \%, 70 \%$ and $96 \%$, for 15, 30 and $30 \mathrm{~min}$, respectively. Pandoravirus massiliensis virions were then

94 placed for $1 \mathrm{~h}$ in a mix of LR-White resin 100\% (Ref. 17411, MUNC-500; Polysciences) and ethanol

$9596 \%$ in a 2:1 ratio. After $30 \mathrm{~min}$ in pure $100 \% \mathrm{LR}-$ White resin, particles were placed in $100 \%$ LR-

96 White resin overnight at room temperature. Pandoravirus particles were then placed for $1 \mathrm{~h}$ in $100 \%$

97 resin at room temperature. A total of $1.5 \mathrm{~mL}$ of Pure $100 \%$ LR-White resin was added on the virus

98 pellet. Polymerization was achieved at $60^{\circ} \mathrm{C}$ for 3 days. Between all steps of inclusion, the samples

99 were ultracentrifuged at 2,400 g and the supernatant was discarded. Sections of 70 or $300 \mathrm{~nm}$-thick

100 were cut on a UC7 ultramicrotome (Leica). Ultrathin sections were deposited on 300 mesh

101 copper/rhodium grids (Maxtaform HR25, TAAB). They were post-stained with 5\% uranyl acetate

102 and lead citrate according to the Reynolds method (Reynolds, 1963). Gold nanoparticles with a

103 diameter of $10 \mathrm{~nm}$ (Ref.752584; Sigma-Aldrich) were deposited on both faces on the $300 \mathrm{~nm}$ thick

104 ultrathin sections for tomographic fiducial alignment. Electron micrographs were acquired on a

105 Tecnai $\mathrm{G}^{2}$ transmission electron microscope operated at $200 \mathrm{keV}$ and equipped with a $4096 \times 4096$

106 pixel resolution Eagle camera (FEI). Tomography tilt series were acquired with the Explore 3D (FEI) 
107 software for tilt ranges of $110^{\circ}$ with $1^{\circ}$ increments. The mean applied defocus was $-4 \mu \mathrm{m}$. The

108 magnification ranged between 9,600 and 29,000 with pixel sizes between 1.12 and $0.37 \mathrm{~nm}$,

109 respectively. The average thickness of the tomograms obtained was $155 \pm 43 \mathrm{~nm}(\mathrm{n}=8$ measures).

110 The tilt-series were aligned using ETomo from the IMOD software package (University of Colorado,

111 USA) (Kremer et al., 1996) by cross-correlation. The tomograms were reconstructed using the

112 weighted-back projection algorithm in ETomo from IMOD (Kremer et al., 1996). ImageJ software

113 was used for image processing (Schneider et al., 2012).

\section{Cellulose staining and light microscopy}

115 Calcofluor staining was achieved by depositing a drop of purified pandoravirus particles in PAS

116 medium onto a glass slide and immediately adding 50 $\mu \mathrm{L}$ of calcofluor white (Ref.18909; Sigma-

117 Aldrich) and $50 \mu \mathrm{L}$ of $10 \% \mathrm{KOH}$ prior to glass slide covering and confocal imaging. Pandoravirus

118 particles were imaged with a Plan-Aprochromat x63/1.4 immersion objective on a AiryScan LSM800

119 confocal laser scanning microscope (Zeiss). Image size was 512x512 pixels and scan zoom ranged

120 from $\mathrm{x} 0.5$ to $\mathrm{x} 2.9$. Laser excitation with a $405 \mathrm{~nm}$ wavelength was used for calcofluor staining

121 imaging and was coupled to an ESID detector for depicting particles contours.

\section{Enzymatic treatment of Pandoravirus massiliensis virions}

123 A total of $50 \mu \mathrm{L}$ of purified virions was added to $1 \mathrm{~mL}$ of cellulase solution (cellulase from

124 Trichoderma reesei, aqueos, Sigma Aldrich, C2730-50ML) at different concentrations and incubated

125 for $48 \mathrm{~h}$ at $45^{\circ} \mathrm{C}$. In a second time, these samples were imaged on AiryScan LSM800 microscope

126 confocal laser scanning microscope. The effects were then observed by confocal microscopy

127 (AiryScan LSM800), scanning microscopy and transmission electronic microscopy. The number of

128 intact viral particles was estimated using the imageJ software (Schneider et al., 2012) 
129 Bioinformatic analyses to search for cellulose synthase candidate genes in the Pandoravirus

130 massiliensis genome

131 Sequences of the predicted ORFs of Pandoravirus massiliensis in amino acids were used for BLAST

132 searches against the NCBI GenBank nr database. The analyses were performed using 1e-2, 25\% and

$13350 \%$ as thresholds for the evalue, the homology and the coverage of aligned sequences, respectively.

134 Phylogenetic reconstruction was performed using the Maximum Likelihood method with the

135 MEGA6 software (Tamura et al., 2013). Conserved domains were also searched for by DELTA-

136 BLAST analyses against the Conserved Domain Database (Marchler-Bauer et al., 2017).

137 BLASTp, tBLASTn and BLASTn analyses were also performed against the gene contents and

138 complete genomes of the 9 other pandoraviruses, i.e. Pandoravirus dulcis, Pandoravirus salinus

139 (Philippe et al., 2013), Pandoravirus inopinatum (Scheid, 2016; Scheid et al., 2014), Pandoravirus

140 quercus (Legendre et al., 2018), Pandoravirus macleodensis (Legendre et al., 2018), Pandoravirus

141 neocaledonia (Legendre et al., 2018), Pandoravirus celtis (Legendre et al., 2019), Pandoravirus

142 braziliensis and Pandoravirus pampulha (Aherfi et al., 2018).

143 Transcription of candidate gene of cellulose synthase of Pandoravirus massiliensis and cellulose 144 synthase of Acanthamoeba castellanii

145 RNA was extracted with the RNeasy mini kit (Cat No: 74104, Qiagen, France) at different time

146 points of the Pandoravirus massiliensis cycle, from H0 (i.e. 45 min after the inoculation of amoeba

147 cells by viral particles) until H12 post-infection (release of neo-synthetized virions). Total RNA was

148 eluted in $50 \mu \mathrm{L}$ of RNase-free water; $2.5 \mu 1$ of RNaseOUT (Thermo Fisher Scientific, France) was

149 added to the eluate to discard RNase. The DNase treatment was performed with TURBO DNase

150 (Invitrogen, France; six cycles of 30 min incubation at $35^{\circ} \mathrm{C}$ ). Two PCR systems targeting the DNA

151 polymerase gene of Pandoravirus massiliensis (forward primer: 5'-ATGGCGCCCGTCTGGAAG; 
reverse primer: 5'-GGCGCCAAAGTGGTGCGA) and the housekeeping gene of the RNA

153 polymerase of Acanthamoeba castellanii (forward: 5'-ACGAACTTCCGAGAGATGCA; reverse: 5'-

154 CACCTTGACCAGTCCCTTCT) were used to check for genomic DNA contamination and as

155 positive controls for the reverse transcription. Primers targeting the candidate gene for the putative

156 cellulose synthase of Pandoravirus massiliensis (forward: 5' TCCACTCGACATGCAATCTT;

157 reverse: 5'-AAAACACAAACCCGCTCTGC) and those targeting the cellulose synthase genes

158 (KC466026.1 and XM_004335119.1) of Acanthamoeba castellanii

159 (forward:5'GGGAGATCAACGACAACCTG; reverse: 5'-GTCCTCRGTCTGCGACTCGT ) were

160 designed using Primer3 (Koressaar and Remm, 2007). RNeasy MinElute Cleanup Kit (Qiagen) was

161 used to purify total RNA according to the manufacturer's recommendations. Total RNA was reverse-

162 transcribed into cDNA by using the SuperScript VILO Synthesis Kit (Invitrogen, France). Then,

163 qPCR was carried out on the cDNA with the LightCycler® 480 SYBR Green 1 Master reaction mix

164 (Roche Diagnostics, Mannheim, Germany), following the manufacturer's temperature program with

$16562^{\circ} \mathrm{C}$ as primer hybridization and elongation temperature. Each experiment was performed in

166 triplicate.

167 Results

168 Pandoravirus massiliensis ultrastructure

169 The study by transmission electron microscopy (TEM) of the ultrastructure of negatively stained

170 purified Pandoravirus massiliensis particles showed ovoid shaped virions with a mean maximal

171 diameter of $1,230 \pm 179 \mathrm{~nm}$ and a mean minimal diameter of $689 \pm 114 \mathrm{~nm}(\mathrm{n}=10)$, these dimensions

172 reaching up to $1,510 \mathrm{~nm}$ x $860 \mathrm{~nm}$ (Figure 1.A). An ostiole with a concave shape could be observed

173 at one apex of the particles (Figure 1.A) and thin fibrillar structures were present around the particles.

174 Fixation with ruthenium red allowed good visualization of peripheral polysaccharides on ultrathin 
175 sections. We observed for all particles, from periphery to inside (Figure 1.B,C): (i) peripheral sugars

176 as depicted by ruthenium red aggregates, with electron-dense spikes originating from a thick layer of

177 electron-dense aggregates ; (ii) an electron-lucent space; (iii) an electron-dense membrane; (iv) an

178 homogeneous interspace; (v) a thick electron-dense layer made of several tubules; (vi) a smooth

179 internal compartment, more dense at each apex. Particles were cut along different planes, thus

180 showing their different orientations, and the ostioles could be observed cut transversally or

181 perpendicularly (Figure 1.D). Structures originating from the thick inner tubular layer could be

182 observed, such as thick tubules reaching the thin outer electron-dense membrane (Figure 1.E) or thin

183 fibrils reaching the most peripheral ruthenium-red stained polysaccharides (Figure 1F).

184 Next, to get a more detailed ultrastructure, a three-dimensional (3D) electron tomography on $300 \mathrm{~nm}$ -

185 thin sections of ruthenium-red fixed LR-White embedded Pandoravirus massiliensis particles was

186 performed. Eight tomograms were reconstructed. A tilt-series acquired at x14,500 magnification

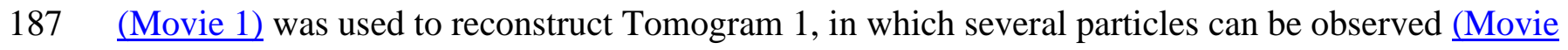

188 2). These particles were ovoid in shape with a homogeneous internal compartment, or non-ovoid with

189 an electron-luscent internal compartment, suggesting deteriorated particles. Tomogram 2 (Movie 3) is

190 a zoom-in on a particle from Tomogram 1. Selected Z-planes from tomogram 2 (Figure 2) illustrate

191 structures originating from the inner thick tubular layer: thick tubular structures with various

192 diameters (black arrows in Figure 2) and thinner tubular/membranous structures of 2 nm-thick at the

193 the ostiole (white arrows in Figure 2). Tomogram 3 is shown in Movie 4. Selected Z-planes from

194 tomogram 3 in Figure 3 show that the inner-most thick tubular layer is composed of two tubules with

195 a diameter of $8 \mathrm{~nm}$ (Figure 3.B). Thin $2 \mathrm{~nm}$-thick fibrils originate from the tubular layer, crossing the

196 electron-dense outer membrane and projecting toward the peripheral sugars (Figure 3.C), or oriented

197 toward the internal compartment on the opposite side of the ostiole (Figure 3D). Tomogram $4 \underline{\text { (Movie }}$

1985 and Movie 6) was chosen to illustrate the continuity between the two tubules from the inner-most 
tubular layer. These tubules can form a U-shape with no ending at the ostiole (Figure 4.A). In

200 addition, we found out that the two inner-most tubules can present a helicoidal arrangement. This

201 finding is shown in tomogram 5 (Movie 6), which is a zoom-in sub-tomogram from tomogram 4. In

202 Figure 4.B, the two tubules from tomogram 5 are arranged as a helix, with locations where the two

203 tubules are distant and other locations where the two tubules are crossed. This helical structural

204 arrangement of the inner-most thick tubular layer then became obvious when playing all tomograms

205 acquired, and this organization could also be noticed when looking back at conventional ultra-thin

206 sections without tomography. The two tubules were superposed forming a $10 \mathrm{~nm}$-thick layer or

207 alternatively distant of $30 \mathrm{~nm}$. On average, this helical arrangement had a periodicity of $150 \mathrm{~nm}$, from

208 crossing points to the most distant location point of the tubules.

209 Subsequently, since the diameter and structure of inner-most tubules potentially forming helix

210 resembled cellulose and chitin according to the literature, we hypothesized thatthat the inner-most

211 layer of the Pandoravirus massiliensis particles was consisted of cellulose and/or chitin.

\section{Cellulose staining}

213 In order to check for cellulose content in Pandoravirus massiliensis particles, a calcofluor white

214 staining of viral particles smears and confocal imaging were performed. Negative control consisting

215 in unstained particles did not show any fluorescence under UV illumination (Figure 5.A, B).

216 Inversely, calcofluor-stained particles were fluorescent under the same illumination (Figure 5.C, D).

217 Single in-liquid Pandoravirus massiliensis particles were stained by calcofluor, as well as particles

218 that remained in a few amoebae still present after purification (Figure 6.A). Zooming on individual

219 particles showed that, on average, the fluorescence of the periphery of the particles seemed to be

220 more intense than in the central region of the same particles (Figure 6.B, C). 
Degradation of the pandoravirus particles by the cellulase treatments and ultrastructure of

\section{cellulase-treated Pandoravirus massiliensis particles}

223 The mean number of viral particles per microscopic field observed after cellulase treatment at high

224 concentrations (stock solution and 1:10 dilution) by confoncal microscopy and assessed on 10

225 microscopic fields by the ImageJ software decreased in comparison with the negative control

226 composed of the same viral sample not submitted to the enzymatic treatment (Figures 7,8 ). With a

227 cellulase solution diluted at 1:10, the number of viral particles was slightly divided by two, and only

$228 \approx 6 \%$ of the virions remained intact after a treatment by the cellulase stock solution. Moreover,

229 calcofluor staining on viral particles treated by cellulase did not show any fluorescence under UV

230 illumination (Figure 9. A1). Besides, a treatment by 1:10 diluted cellulase solution showed a

231 fluorescent matrix (Figure 9.B1) suggesting a degradation of a cellulosic part of the viral particles. It

232 should be noted that this result was less obvious in the most diluted solution of the enzyme (1:100)

233 (Figure 9.C1). Furthermore, the scanning microscopy of pandoravirus virions treated with cellulase at

234 high concentration confirmed the progressive degradation of particles (Figures 9.A1 to D3). It also

235 showed the partial digestion of pandoravirus particles treated with cellulase at 1:10 dilution, by

236 forming a matrix (Figures 9.B2,B3).

As compared to control, untreated Pandoravirus massiliensis particles (Figure 10.A1-A5), a

238 degradation of the virions by the cellulase solution was observed by transmission electron

239 microscopy (Figure 10.B-D). Albeit this effect was not homogeneous between particles in all

240 conditions, defective particles were observed with a dose-dependent effect of cellulase at the

241 peripheral envelope and/or the internal compartment. Indeed, at 1/100 cellulase concentration,

242 particles showed a defect of the envelope (Figure 10.B3-B5) with the presence of electron-luscent

243 regions between the thin electron-dense membrane and the thick bundle of tubules described in

244 Figure 1. Detachments from the different layers were also observed in most of the affected particles. 
(Figure 10.B5). Accordingly, with 1/10 cellulase concentration treatment, particles exhibited

electron-luscent regions at the level of the envelope as well as detachments of the different layers

247 (Figure 10.C3-C5), and also defects in the internal compartment with empty spaces and vacuoles

248 (Figure 10.C4,C5). This defect in the internal compartment was even more observable at stock

249 concentration of cellulase (Figure 10.D2-D4), with 'ghost-like' particles presenting totally empty

250 internal spaces and only a thin surrounding envelope (Figure 10.D5). We also noticed the presence of

251 debris and/or of an amorphous matrix in the ultra-thin sections of cellulase-treated pandoravirus

252 virions (Figure 10.B1,C1,D1), in a dose-dependent manner coherent with the amorphous matrix seen

253 by light microscopy and scanning electron microscopy under the same conditions (Figure 10), which

254 may correspond to the debris of cellulase-digested particles.

\section{Cellulose synthase candidate gene in the Pandoravirus massiliensis gene content}

DELTA-BLAST analyses revealed a distant homology for the predicted gene 594 of Pandoravirus

massiliensis with the cellulose synthase domain bcsA of different bacteria such as Escherichia coli,

Shigella sp., Salmonella enterica for the 30 best hits. These homologies were barely significant with

259 the 10 best hits, identity percentages in amino acids varying between $24.7 \%$ and $23.9 \%$, query

260 coverages between $24 \%$ and $25 \%$, and e-values ranging between 2e-15 and 3e-15 (Supplementary

261 File 1). For this ORF594, no homology with a cellulose synthase was found by BLAST analyses

262 neither against the nr database, nor against the conserved domain database (CDD) (Marchler-Bauer et

263 al., 2017). Orthologs of this ORF594 were found by BLASTp analyses in all the 9 other

264 pandoraviruses, with e-values varying from 3.41e-11 to 0.006; but none of them harbored the same

265 bcsA domain when searched for by DELTA-BLAST analysis or in the Conserved Domain Database.

266 Transcription of candidate gene of cellulose synthase of Pandoravirus massiliensis and cellulose 
All qPCR experiments performed on $P$. massiliensis DNA using primers targeting the cellulose

synthase candidate gene (ORF594) were positive, whereas all those performed on A. castellanii Neff

DNA using the same primers were negative, which confirmed the specificity of the PCR system.

271 Conversely, qPCR targeting the cellulose synthase gene of A. castellanii was positive on the amoebal

272 DNA and negative on the viral DNA, confirming the amoebal specificity of these primers. All qPCR

273 tests performed on the purified RNA extract after DNase treatment before the reverse transcription

274 step were negative, indicating efficient DNA degradation.

qRT-PCR targeting $P$. massiliensis ORF594 were positive on the viral cDNA for samples collected

276 during the whole viral cycle, indicating that this gene was transcribed. qRT-PCR targeting $A$.

277 castellanii cellulose synthase gene were positive on the cDNA obtained from uninfected amoebae

278 and from amoebae infected by $P$. massiliensis during the whole replicative cycle (Figure 11). These

279 latter results show that the cellulose synthase gene of Acanthamoeba castellanii is transcribed both in

uninfected amoebae and in amoebae infected with Pandoravirus massiliensis.

\section{Discussion}

282 We have determined here, by studying the ultrastructure of Pandoravirus massiliensis virions by

283 transmission electron microscopy after various treatments, that its viral integument, not previously

284 characterized, was partially of a polysaccharide nature with a helical structure comparable to that of

285 vegetable cellulose. Several markers of sugars (red rhutenium, calcofluor), the degradation of virions

286 by cellulase and the use of appropriate negative controls clearly confirmed the cellulosic nature of the

287 pandoravirus viral tegument. The cellulose is the most common biologic macromolecule,

288 biosynthesized by vegetals, algae, some bacteria, and by some marine animals as Ascidiae (Chen et

289 al., 2016; Helenius et al., 2006; Nakashima et al., 2004). It is also a major component of the cyst of

290 Acanthamoeba, the unique host of pandoraviruses demonstrated to date (Magistrado-Coxen et al., 
2019). It is composed of polymers of $\beta(1,4)$ glucose subunits. Cellulosic chains are structured as

microfibrils, which confer both resistance and plasticity to the vegetal walls, and also probably to the

293

tegument of Pandoravirus massiliensis virions. The bioinformatic analyses of the Pandoravirus

294 massiliensis genome possibly revealed a candidate gene (ORF594) for a cellulose synthase, which

295 could be involved in the cellulose synthesis of the viral tegument. Indeed, a distant homology with

296 the BcsA domain, which is one of the four catalytic subunits of the bacterial cellulose synthase was

297 found (Wong et al., 1990). The domain bcsA polymerizes 5'-UDP-glucose to the cellulose polymer

298 in formation (Zimmer, 2015). In the 9 other pandoraviruses, an ortholog for the ORF594 was found

299 by BLASTp analysis. The experiments of qRT PCR revealed that this gene was transcribed during

300 the viral cycle with an increased transcription 4, 6 and 8 hours post-infection, the time points

301 matching with the formation of viral factories, which could reinforce this first hypothesis.

302 However, the similarity with the bcsA domain is very low and this domain was not found in none of

303 the 9 other pandoraviruses. Besides, the length of this domain bcsA found in bacteria ranges

304 approximately around 800 amino-acids in length. The ORF594 is slightly smaller with a predicted

305 encoded protein of only 135 amino-acids in length. The 3 other subunits of the cellulose synthase

306 were not found in the Pandoravirus massiliensis genome, neither by BLASTp nor by DELTA Blast

307 analyses. Therefore, although this ORF594 is transcribed during the replicative cycle, the implication

308 of the ORF594 in the synthesis of the cellulose is unlikely. One of the most amazing features of the

309 pandoraviruses is the tremendous proportion of ORFans (genes without any homolog in the

310 international sequence databases) and genes predicted to encode hypothetical proteins in their

311 genome. These recently described viruses are so far to be exhaustively characterized. It has been

312 shown that these hypothetical proteins are transcribed and translated (Aherfi et al., 2018; Legendre et

313 al., 2018; Philippe et al., 2013; Scheid, 2016) suggesting that they have an efficient function for the

314 virus. Among all these predicted genes, some of them could be implied in the synthesis of the 
315 cellulose, i.e. other unidentified cellulose synthase subunits, through synthesis by new enzymes or by

316 a new metabolic pathway, unknown to date.

317 We could alternatively hypothesize that the virus could use for the synthesis of its particles

318 tegument, the gene of the cellulose synthase of Acanthamoeba castellanii (Clarke et al., 2013). In a

319 previous work, it has been shown that when Acanthamoeba was infected with a mimivirus, the

320 transcription of the amoeba felt dramatically and at 6 hours after infection, the transcription became

321 undetectable (Legendre et al., 2010). We observed herein, that the host gene of cellulose synthase

322 was transcribed during the all the replication cycle of Pandoravirus massiliensis, showing that the

323 transcription of this amoebal gene is not impacted by the infection with Pandoravirus. Therefore, we

324 can assume that the amoebal cellulose synthase could be diverted to the virus benefits, and could be

325 involved in the synthesis of the cellulosic viral tegument. This gene was also found as transcribed,

326 while Acanthamoeba castellanii were in the survival buffer, a buffer with the minimal components

327 for the survival of amoebae, and thus leading to the amoebal enkystment. At this time, the amoeba

328 could start the synthesis of cellulose, a main component of the amoebal cyst.

329 In conclusion, we could determine herein the cellulosic nature of the tegument of Pandoravirus

330 massiliensis. Although a distant similarity was found with the catalytic subunit bcsA of the bacterial

331 cellulose synthase, with a predicted ORF of Pandoravirus massiliensis, this domain was not found in

332 any other pandoravirus. These data suggest that the cellulose of the tegument of pandoraviruses could

333 be probably the product of the host amoebal cellulose synthase. These results provide new data

334 information about this important virus and contributes to the understanding of the biology of these

335 complex viruses and their definition and classification in the virosphere. 
Authors contributions

337 DBB and JPB did the experiments and wrote the paper. FDP and FG did the experiments. PC wrote

338 the paper. SA supervised the experiments and wrote the paper. BLS conceived the project, supervised

339 the experiments and wrote the paper.

$341 \quad$ Funding

342 This work was supported by the French Government under the "Investments for the Future" program

343 managed by the National Agency for Research (ANR), Méditerranée-Infection 10-IAHU-03 and was

344 also supported by Région Provence-Alpes-Côte d'Azur and European funding FEDER PRIMMI

345 (Fonds Européen de Développement Régional - Plateformes de Recherche et d'Innovation Mutuali

346 sées Méditerranée Infection).

\section{Conflict of Interest}

349 The authors declare that the research was conducted in the absence of any commercial or financial 350 relationships that could be construed as a potential conflict of interest.

352 The movies for this article can be found online at : https://www.mediterranee-infection.com/acces-

353 ressources/donnees-pour-articles/pandoravirus-massiliensis-tomograms/ 
Aherfi, S., Andreani, J., Baptiste, E., Oumessoum, A., Dornas, F.P., Andrade, A.C.D.S.P., Chabriere, E., Abrahao, J., Levasseur, A., Raoult, D., et al. (2018). A Large Open Pangenome and a Small Core Genome for Giant Pandoraviruses. Front. Microbiol. 9, 1486. doi:10.3389/fmicb.2018.01486. Cedratvirus, a Double-Cork Structured Giant Virus, is a Distant Relative of Pithoviruses. Viruses 8. doi:10.3390/v8110300.

Andreani, J., Khalil, J.Y.B., Baptiste, E., Hasni, I., Michelle, C., Raoult, D., Levasseur, A., and La Scola, B. (2018).

Chen, Y.W., Lee, H.V., Juan, J.C., and Phang, S.-M. (2016). Production of new cellulose nanomaterial from red algae marine biomass Gelidium elegans. Carbohydr. Polym. 151, 1210-1219. doi:10.1016/j.carbpol.2016.06.083. evolution of tyrosine kinase signaling. Genome Biol. 14, R11. doi:10.1186/gb-2013-14-2-r11. viruses. Arch. Virol. 158, 2517-2521. doi:10.1007/s00705-013-1768-6.

Koressaar, T., and Remm, M. (2007). Enhancements and modifications of primer design program Primer3. Bioinforma. Oxf. Engl. 23, 1289-1291. doi:10.1093/bioinformatics/btm091.

Kremer, J.R., Mastronarde, D.N., and McIntosh, J.R. (1996). Computer visualization of three-dimensional image data using IMOD. J. Struct. Biol. 116, 71-76. doi:10.1006/jsbi.1996.0013. Raoult, D. (2003). A giant virus in amoebae. Science 299, 2033. doi:10.1126/science.1081867.

Legendre, M., Audic, S., Poirot, O., Hingamp, P., Seltzer, V., Byrne, D., Lartigue, A., Lescot, M., Bernadac, A., Poulain, J., et al. (2010). mRNA deep sequencing reveals 75 new genes and a complex transcriptional landscape in Mimivirus. Genome Res. 20, 664-674. doi:10.1038/s41467-018-04698-4.

Legendre, M., Bartoli, J., Shmakova, L., Jeudy, S., Labadie, K., Adrait, A., Lescot, M., Poirot, O., Bertaux, L., Bruley, C., et al. (2014). Thirty-thousand-year-old distant relative of giant icosahedral DNA viruses with a pandoravirus morphology. Proc. Natl. Acad. Sci. U. S. A. 111, 4274-4279. doi:10.1101/gr.102582.109.

Legendre, M., Fabre, E., Poirot, O., Jeudy, S., Lartigue, A., Alempic, J.-M., Beucher, L., Philippe, N., Bertaux, L., Christo-Foroux, E., et al. (2018). Diversity and evolution of the emerging Pandoraviridae family. Nat. Commun. 9, 2285. doi:10.3389/fmicb.2019.00430. Front. Microbiol. 10, 430. doi:10.1073/pnas.1320670111.

392 Magistrado-Coxen, P., Aqeel, Y., Lopez, A., Haserick, J.R., Urbanowicz, B.R., Costello, C.E., and Samuelson, J. (2019). 
395 Marchler-Bauer, A., Bo, Y., Han, L., He, J., Lanczycki, C.J., Lu, S., Chitsaz, F., Derbyshire, M.K., Geer, R.C., Gonzales, 396 N.R., et al. (2017). CDD/SPARCLE: functional classification of proteins via subfamily domain architectures. Nucleic

397 Acids Res. 45, D200-D203. doi:10.1371/journal.pntd.0007352.

398 Nakashima, K., Yamada, L., Satou, Y., Azuma, J., and Satoh, N. (2004). The evolutionary origin of animal cellulose

399 synthase. Dev. Genes Evol. 214, 81-88. doi:10.1007/s00427-003-0379-8.

400 Pereira Andrade, A.C.D.S., Victor de Miranda Boratto, P., Rodrigues, R.A.L., Bastos, T.M., Azevedo, B.L., Dornas, F.P., 401 Oliveira, D.B., Drumond, B.P., Kroon, E.G., and Abrahão, J.S. (2019). New Isolates of Pandoraviruses: Contribution to

402 the Study of Replication Cycle Steps. J. Virol. 93. doi:10.1128/JVI.01942-18.

403 Philippe, N., Legendre, M., Doutre, G., Couté, Y., Poirot, O., Lescot, M., Arslan, D., Seltzer, V., Bertaux, L., Bruley, C., 404 et al. (2013). Pandoraviruses: amoeba viruses with genomes up to $2.5 \mathrm{Mb}$ reaching that of parasitic eukaryotes. Science

405 341, 281-286. doi:10.1126/science.1239181.

406 Reynolds, E.S. (1963). The use of lead citrate at high pH as an electron-opaque stain in electron microscopy. J. Cell Biol. 407 17, 208-212. doi:10.1083/jcb.17.1.208.

408 Scheid, P. (2016). A strange endocytobiont revealed as largest virus. Curr. Opin. Microbiol. 31, 58-62.

409 doi:10.1016/j.mib.2016.02.005.

410 Scheid, P., Balczun, C., and Schaub, G.A. (2014). Some secrets are revealed: parasitic keratitis amoebae as vectors of the 411 scarcely described pandoraviruses to humans. Parasitol. Res. 113, 3759-3764. doi:10.1007/s00436-014-4041-3.

412 Schneider, C.A., Rasband, W.S., and Eliceiri, K.W. (2012). NIH Image to ImageJ: 25 years of image analysis. Nat.

413 Methods 9, 671-675. doi:10.1038/nmeth.2089.

414 Tamura, K., Stecher, G., Peterson, D., Filipski, A., and Kumar, S. (2013). MEGA6: Molecular Evolutionary Genetics

415 Analysis version 6.0. Mol. Biol. Evol. 30, 2725-2729. oi:10.1093/molbev/mst197.

416 Wong, H.C., Fear, A.L., Calhoon, R.D., Eichinger, G.H., Mayer, R., Amikam, D., Benziman, M., Gelfand, D.H., Meade, 417 J.H., and Emerick, A.W. (1990). Genetic organization of the cellulose synthase operon in Acetobacter xylinum. Proc.

418 Natl. Acad. Sci. 87, 8130-8134. doi:10.1073/pnas.87.20.8130.

419 Zimmer, J. (2015). A Molecular Description of Cellulose Biosynthesis. Biophys. J. 108, 499a.

420 doi:10.1016/j.bpj.2014.11.2734. 
FIGURES :
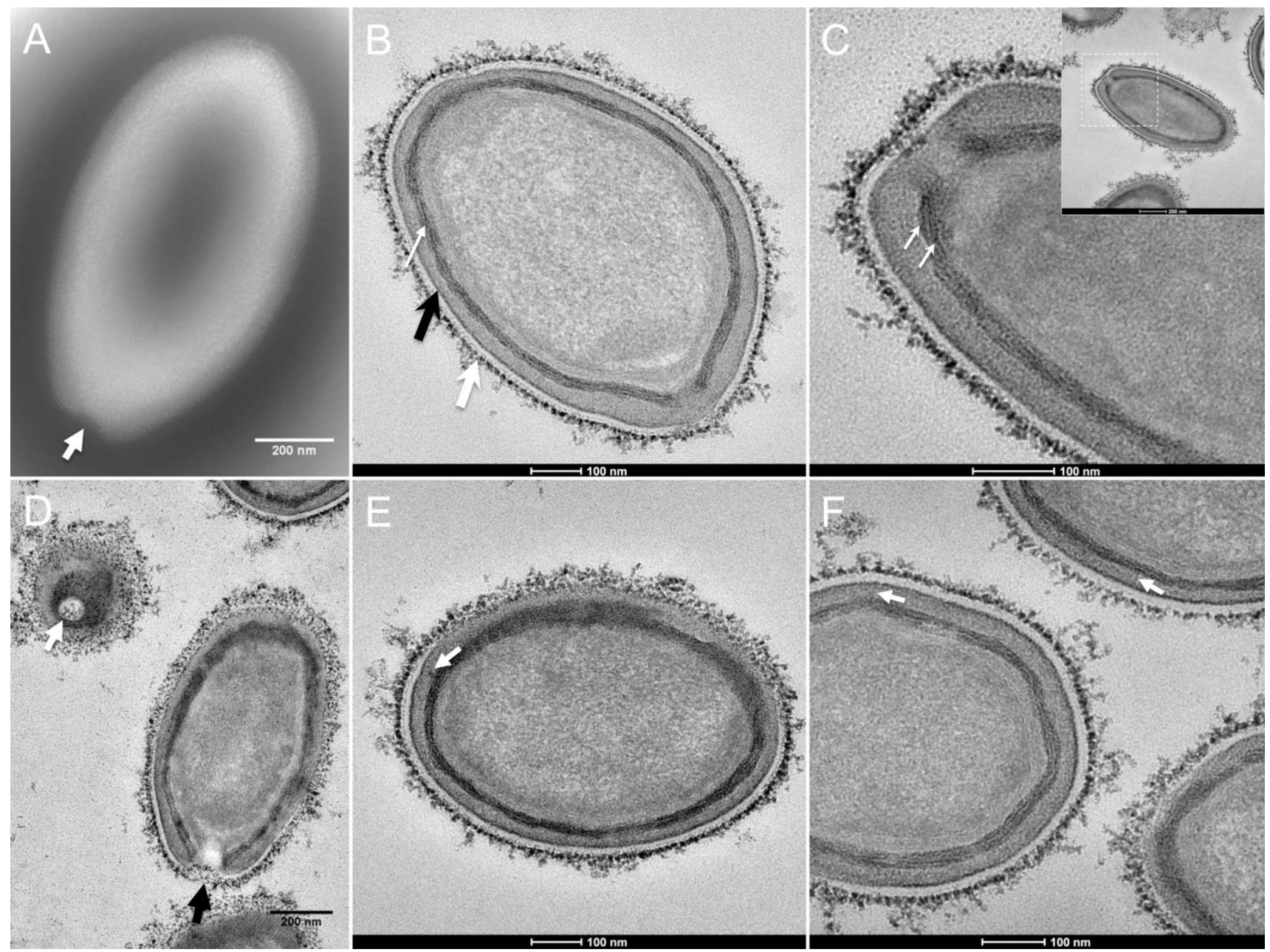

427 Figure 1. Transmission electron microscopy of Pandoravirus massiliensis.

428 A. Negative staining of a Pandoravirus massiliensis particle: the ostiole (arrow) is located at the apex

429 of the particle. Peripheral thin fibers can be observed enwrapping the particle. B. Ultrathin section

430 showing i) the most-peripheral sugars depicted by ruthenium red aggregates (thick white arrow) ; ii) a

431 thin electron-dense membrane (thick black arrow) and more centrally iii) a thick bundle of tubules

432 (thin white arrow). C. Two thick tubules compose the inner-most thick layer. D. Two particles with

433 ostioles cut transversally or perpendicularly. E. The inner-most thick tubules with thick protrusions

434 toward the outer thin electron-dense membrane. F. Thin fibers projecting from the inner-most thick 

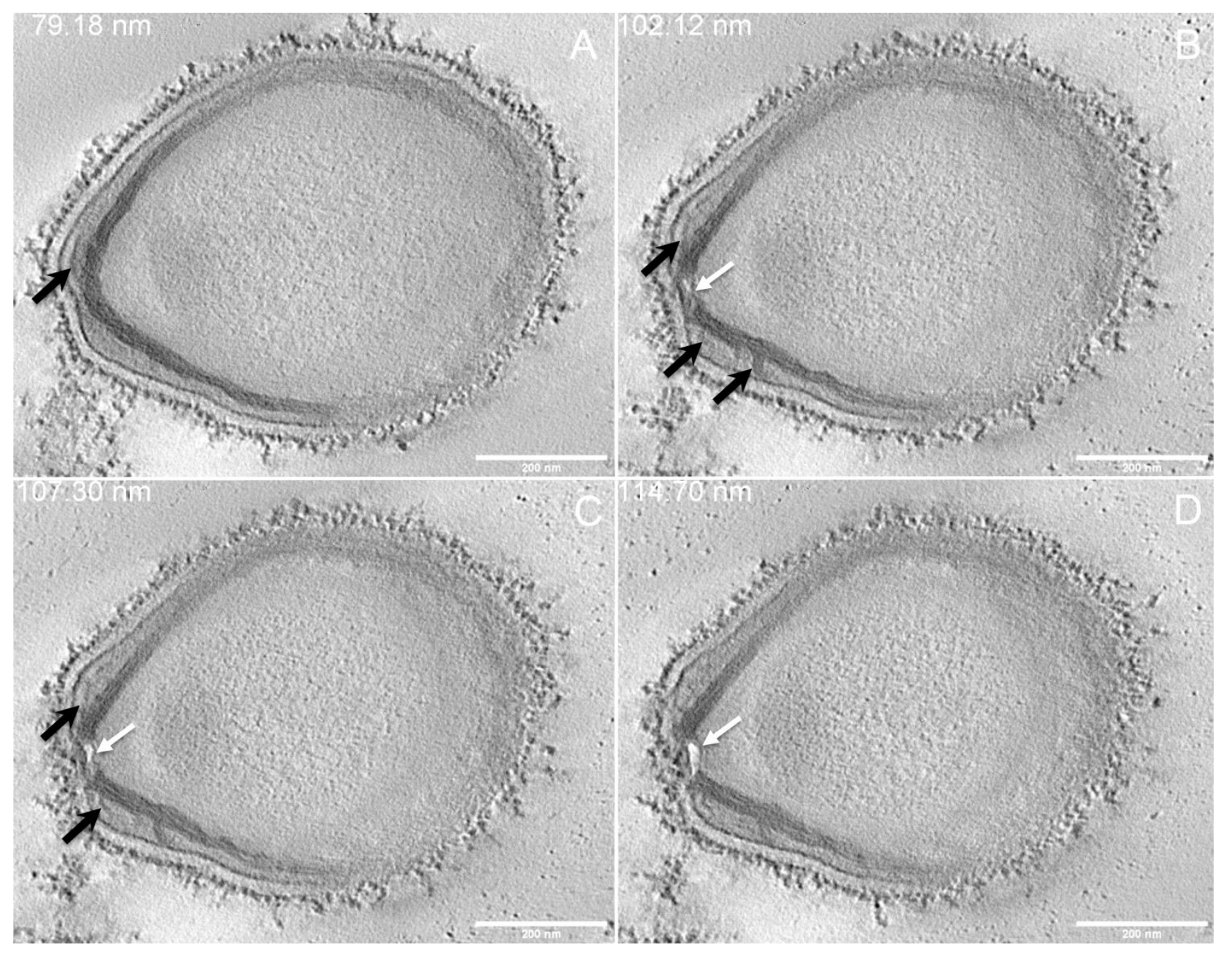

440 A-D. Single planes in the tomogram from Movie3 showing thick tubules protruding toward the

441 periphery and the outer electron-dense membrane (black arrows); a thin tubule/membrane (white 442 arrow) connects the thick tubules layers located on each side of the ostiole. 

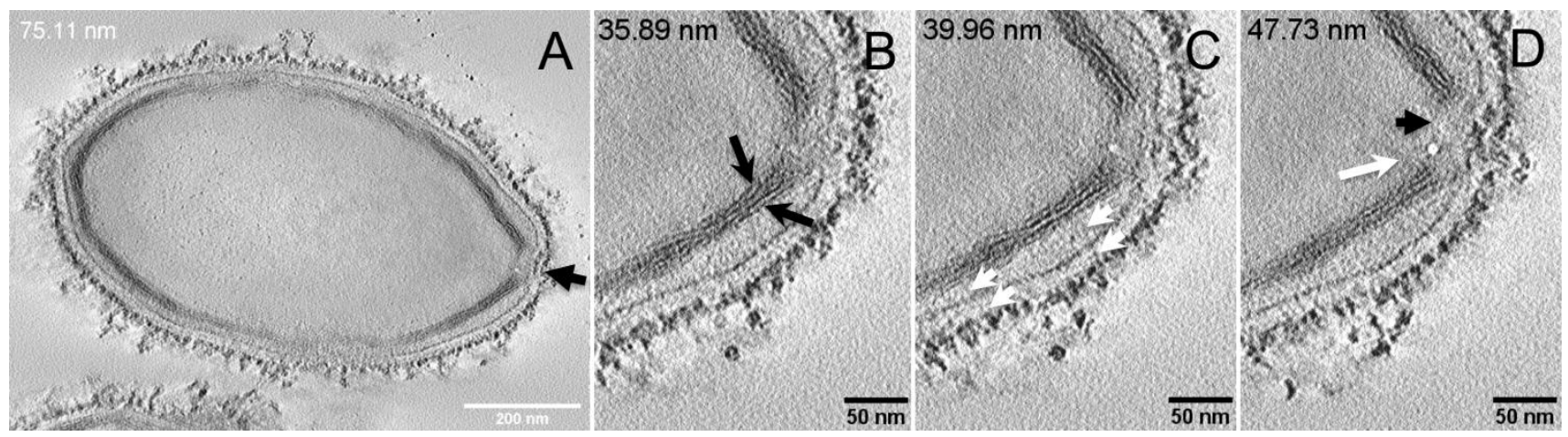

445 Figure 3. Electron tomography of Pandoravirus massiliensis particle from Movie4.

446 A. Single plane in the tomogram from Movie4 showing a whole Pandoravirus particle and its ostiole

447 located at one apex (arrow). B. The inner-most layer is composed of two thick tubules well separated

448 as seen here or contacting each other. C-D. Thin fibers (white arrows) originating from the inner-

449 most tubular thick layer projecting toward the peripheral sugars (white arrows, C), toward the inner

450 core of the particle (white arrow, D) or at the level of the ostiole (black arrow, D). 

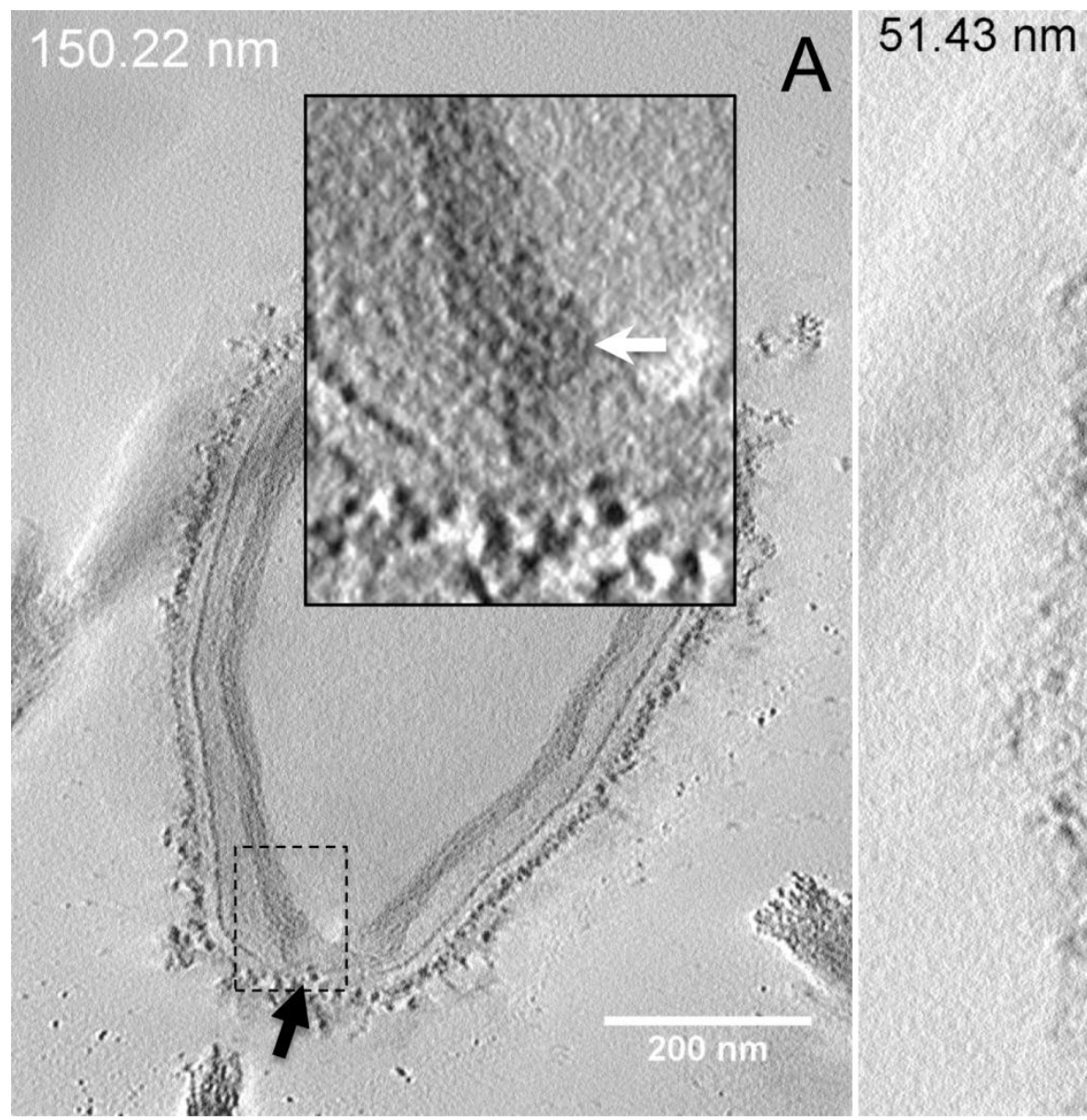

Figure 4. Electron tomography of Pandoravirus massiliensis particles from Movies 5 and 6.

454 A. Single plane in the tomogram from Movie5 showing a Pandoravirus particle with its ostiole (black

455 arrow). The magnified boxed region depicts a U-shaped thick tubule from the inner-most layer. B.

456 Single plane in Movie6 from the zoomed-in tomogram from Movie5 showing the helical structural

457 arrangement of the two thick tubules (black arrows) composing the inner-most layer of Pandoravirus 458 particles. 


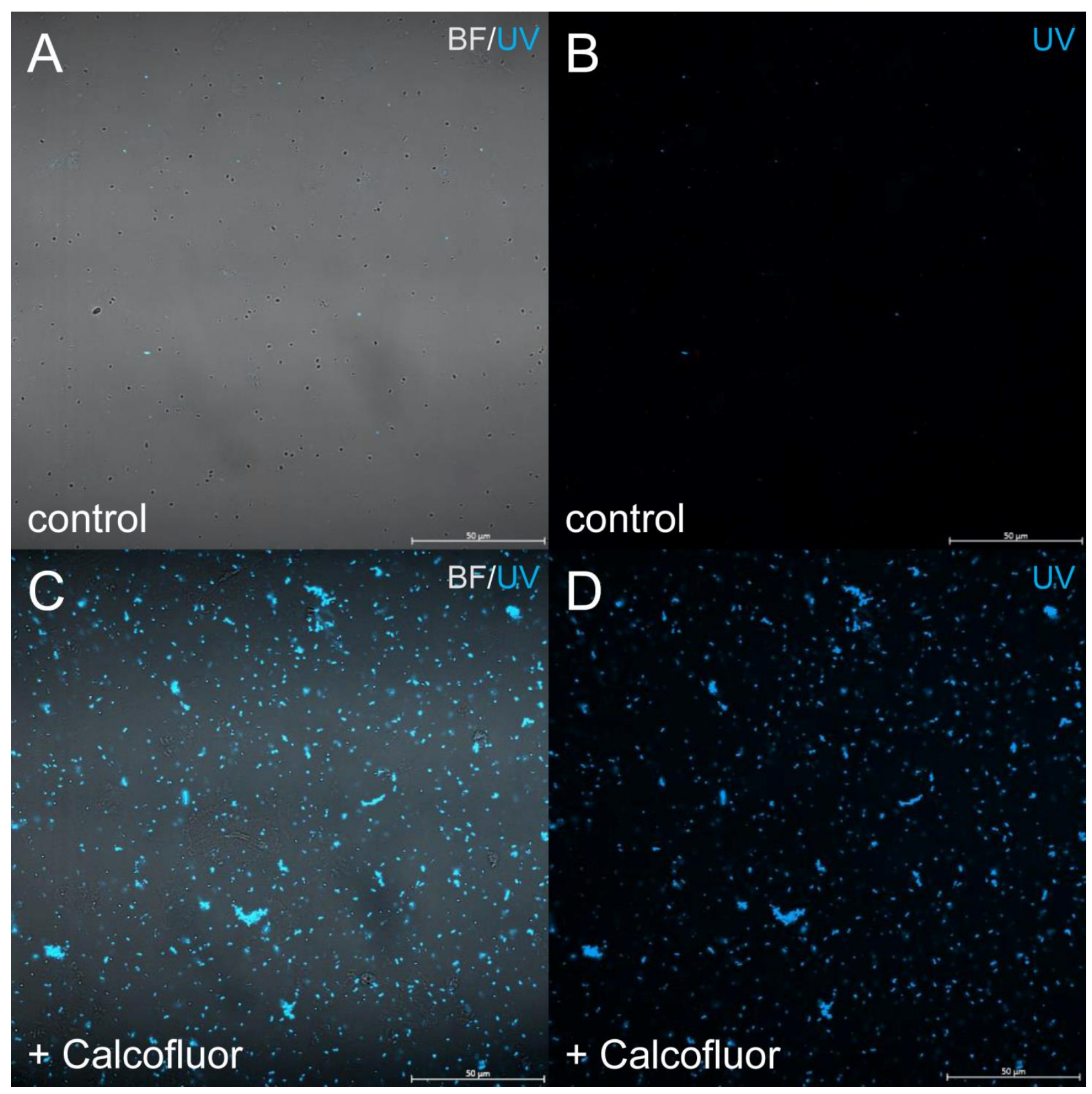

461 Figure 5. Confocal imaging of Calcofluor staining of Pandoravirus massiliensis.

462 A,B. Control Pandoravirus particles. C,D. Calcofluor-stained Pandoravirus particles. 

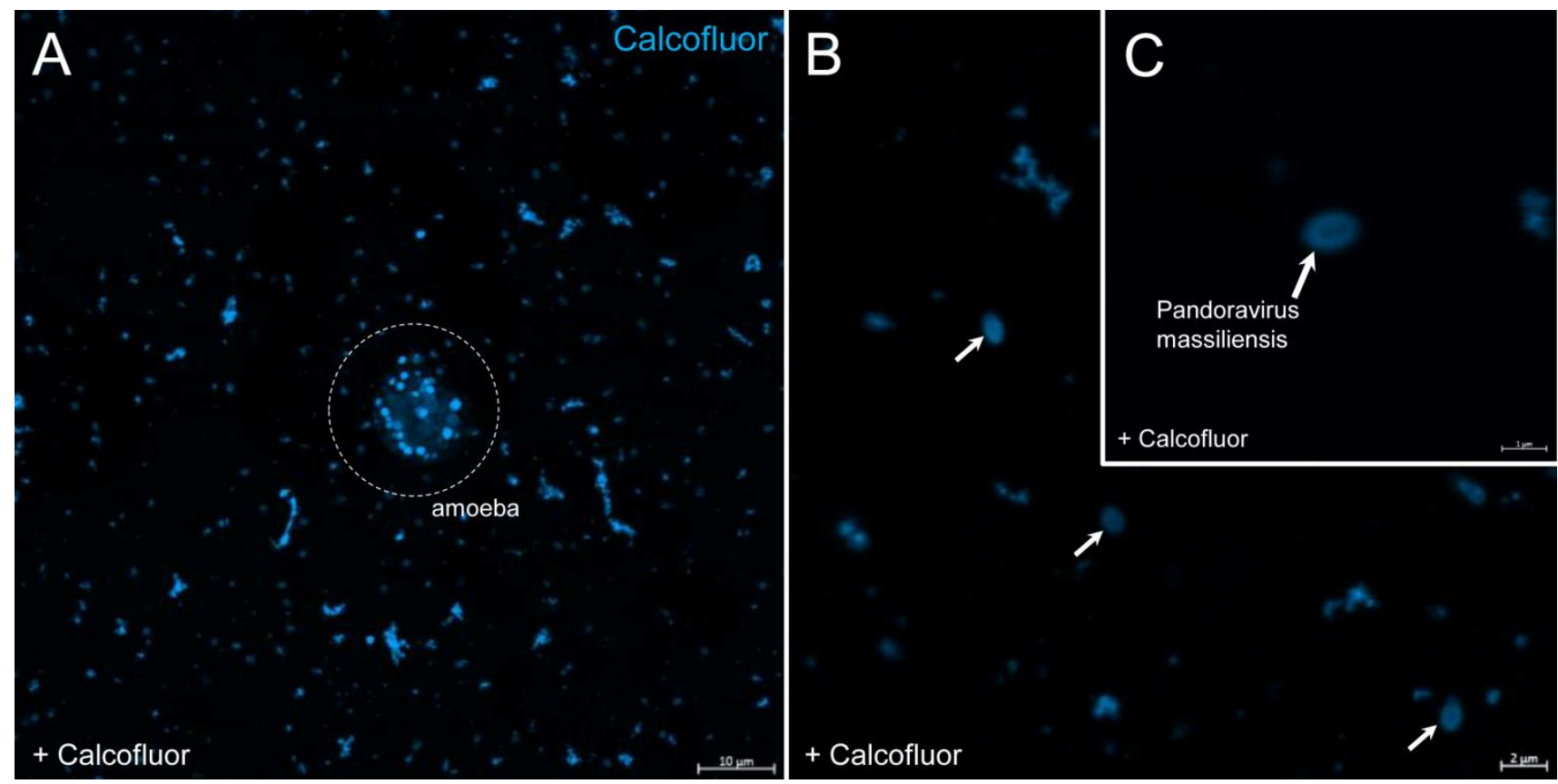

465 Figure 6. Confocal imaging of Calcofluor staining of Pandoravirus massiliensis.

466 A. Pandoravirus-infected amoeba and single Pandoravirus particles stained with Calcofluor white.

467 B,C. Calcofluor-stained Pandoravirus particles showing an intense peripheral calcofluor signal and a

468 less-stained central region.
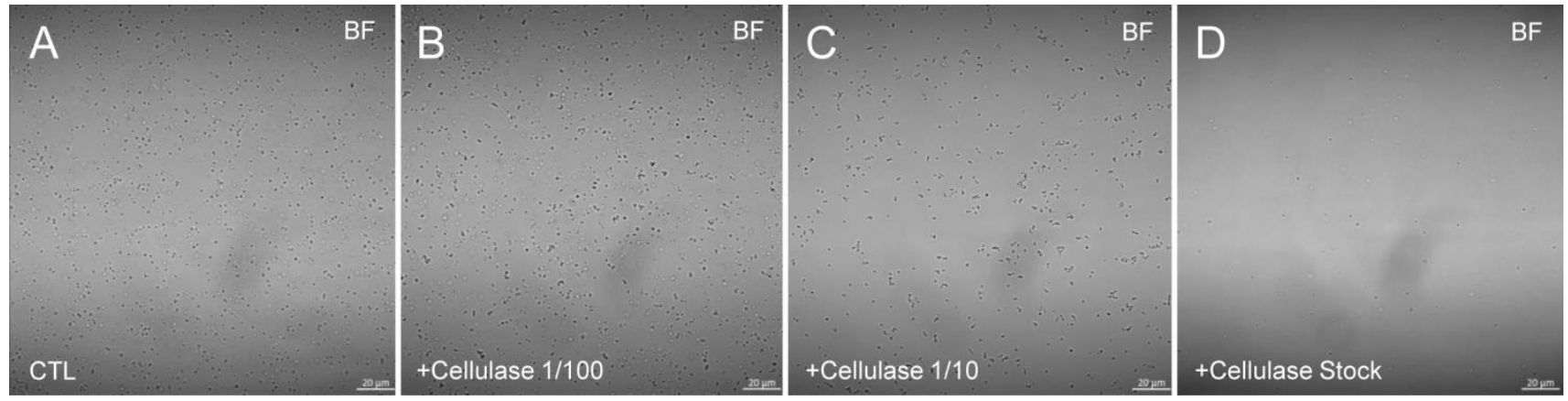

471 Figure 7. Confocal imaging of cellulase-treated Pandoravirus massiliensis.

472 A. Control condition with untreated Pandoravirus massiliensis particles. B-E: cellulase-treated 473 Pandoravirus massiliensis particles. 


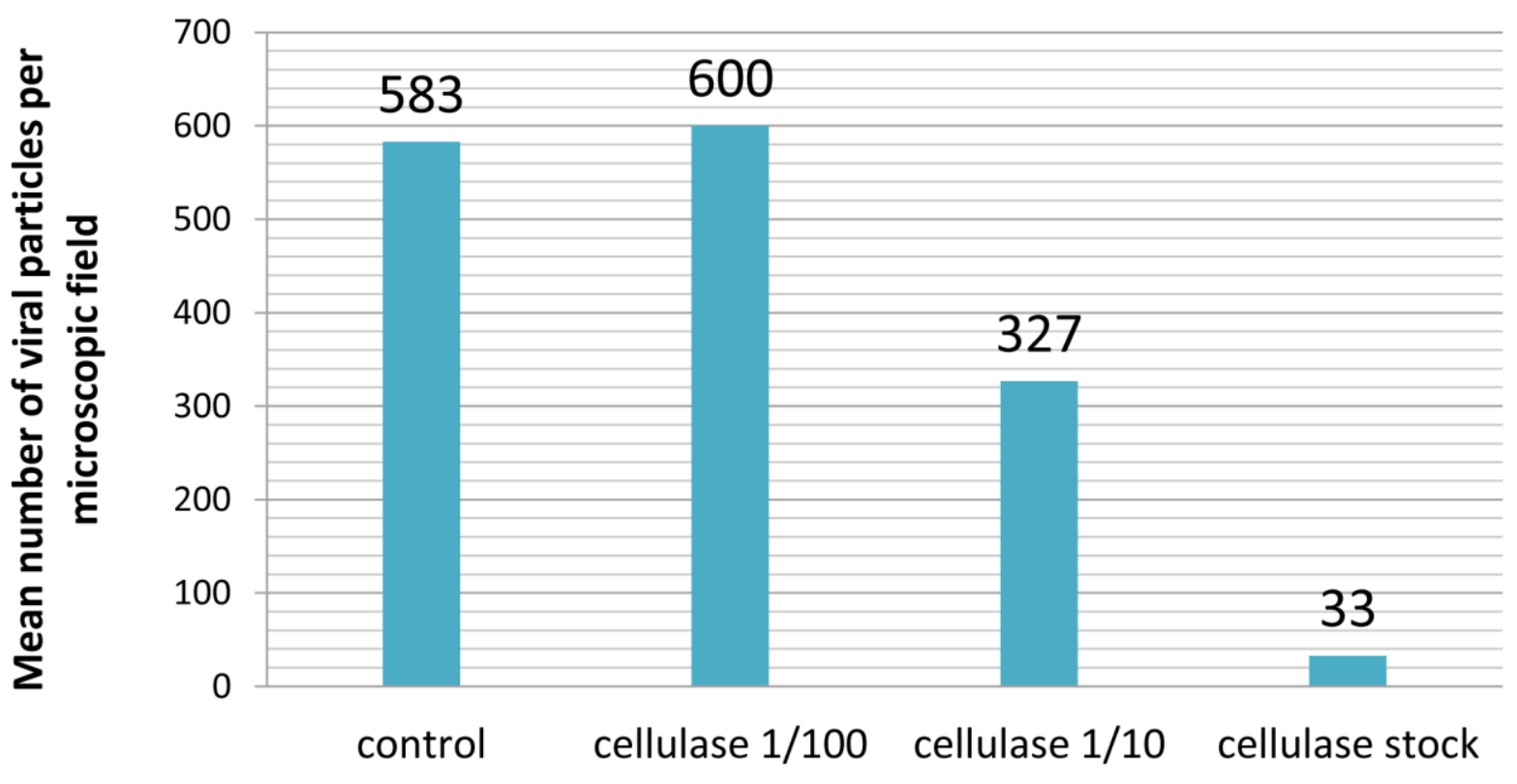

Cellulase concentration

477 Figure 8 : Estimation of the mean number of particles of Pandoravirus massiliensis per microscopic

478 field of observation after cellulase treatment, assessed by the imageJ software. 


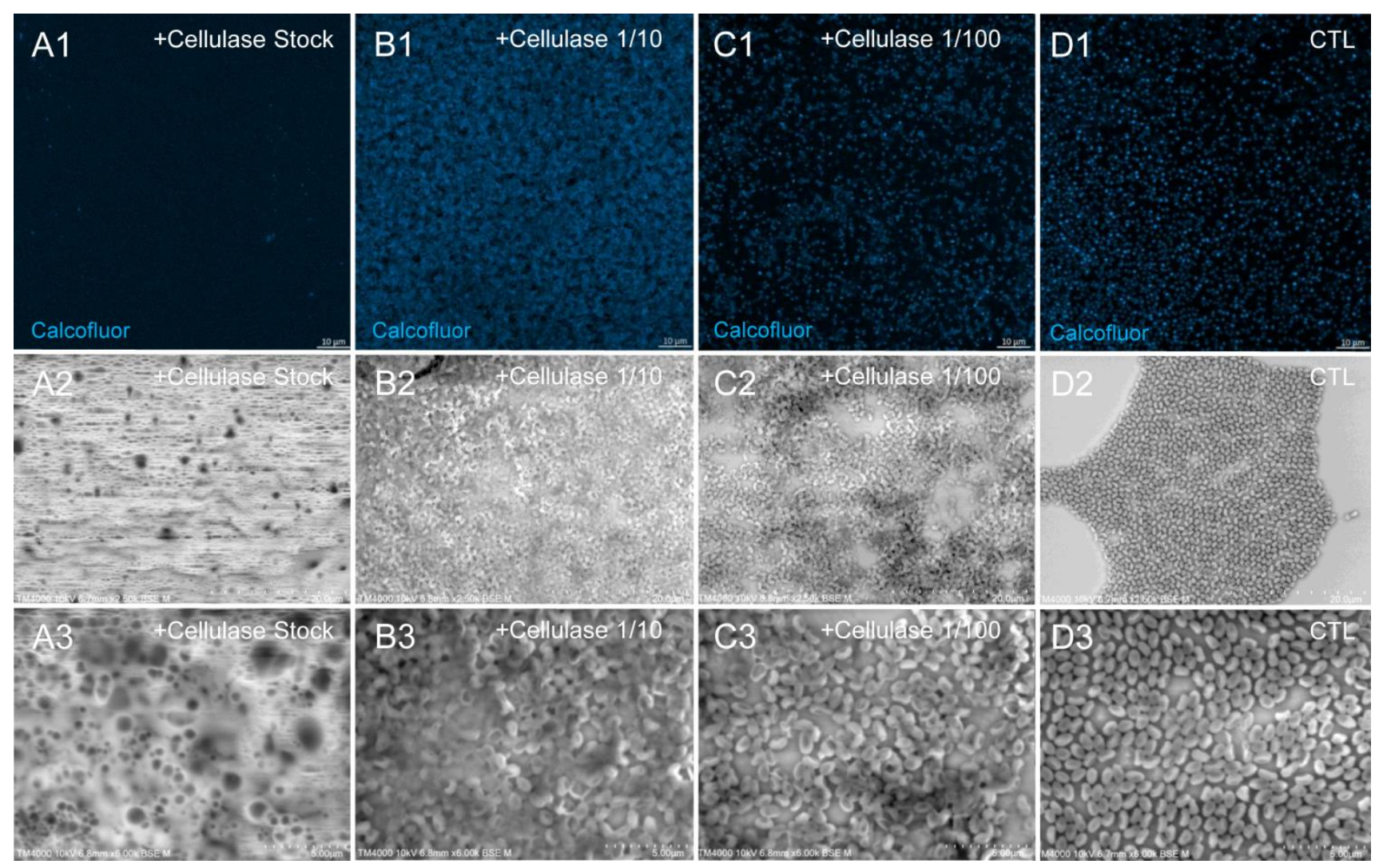

481 Figure 9. Confocal imaging of Calcofluor-stained cellulase-treated Pandoravirus massiliensis

482 virions and scanning microscopy of cellulose treated Pandoravirus massiliensis.

483 A1-C1: cellulase-treated Pandoravirus massiliensis particles stained with Calcofluor-white.D1

484 Control condition with untreated Pandoravirus massiliensis particles stained with Calcofluor-white.

$485 \mathrm{~A}(2-3) \mathrm{B}(2-3)-\mathrm{C}(2-3)$ : cellulase-treated Pandoravirus massiliensis particles.D2.D3 Control condition

486 with untreated Pandoravirus massiliensis .. imaged with scanning microscopy on two magnification. 


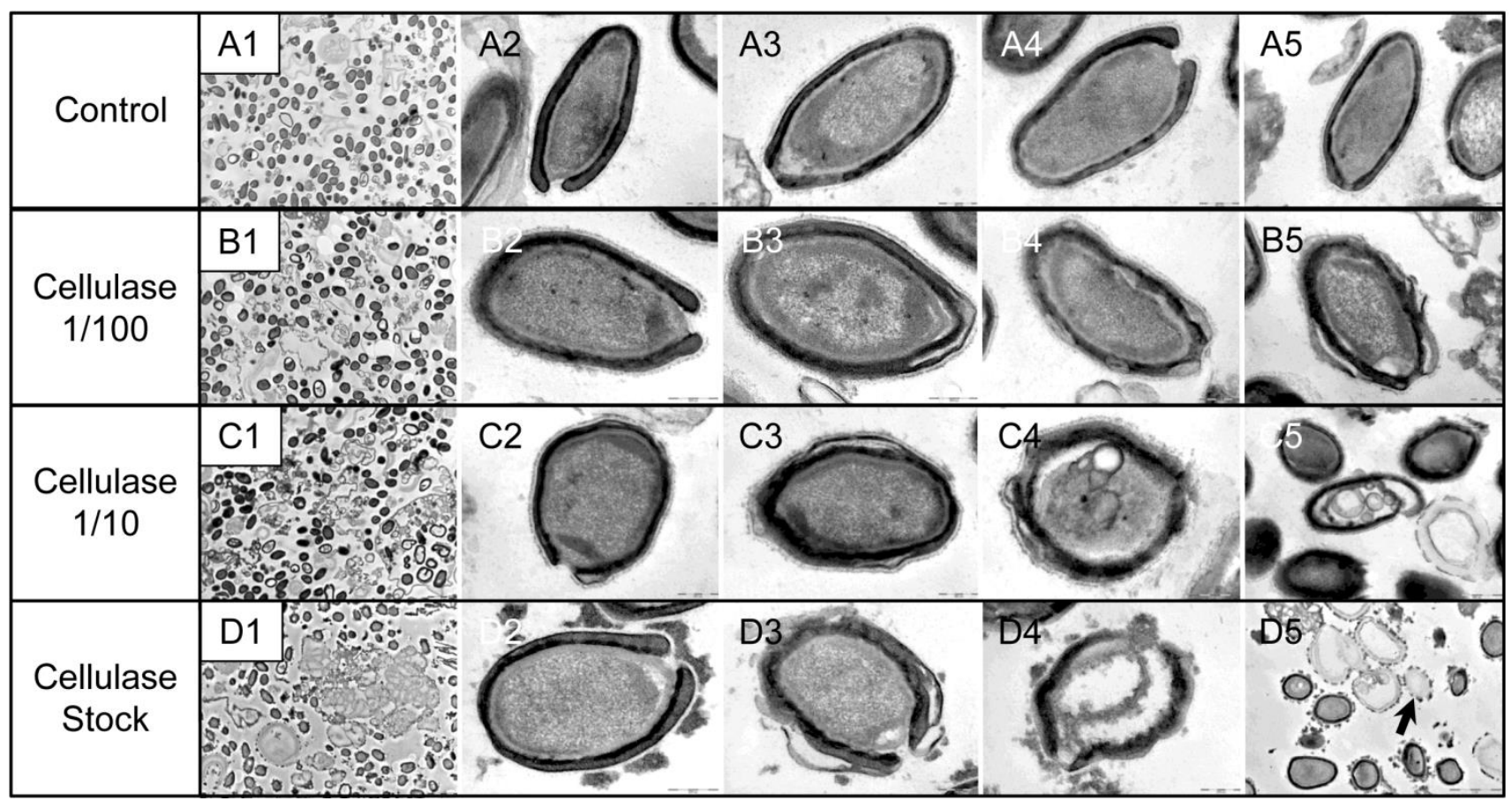

Figure 10. Scanning microscopy of cellulase- treated Pandoravirus massiliensis particles.

490 A1-A4 : Control condition with untreated Pandoravirus massiliensis particles .B-D Cellulase-treated

491 Pandoravirus massiliensis particles imaged in different stages of digestion 1-4 from least to the most

492 digested B.C.D (1): intact particles. B.C.D (2): the beginning of digestion. B.C.D(3): in the middle of

493 digestion. B.C.D (4): at the end of the enzymatic digestion. 


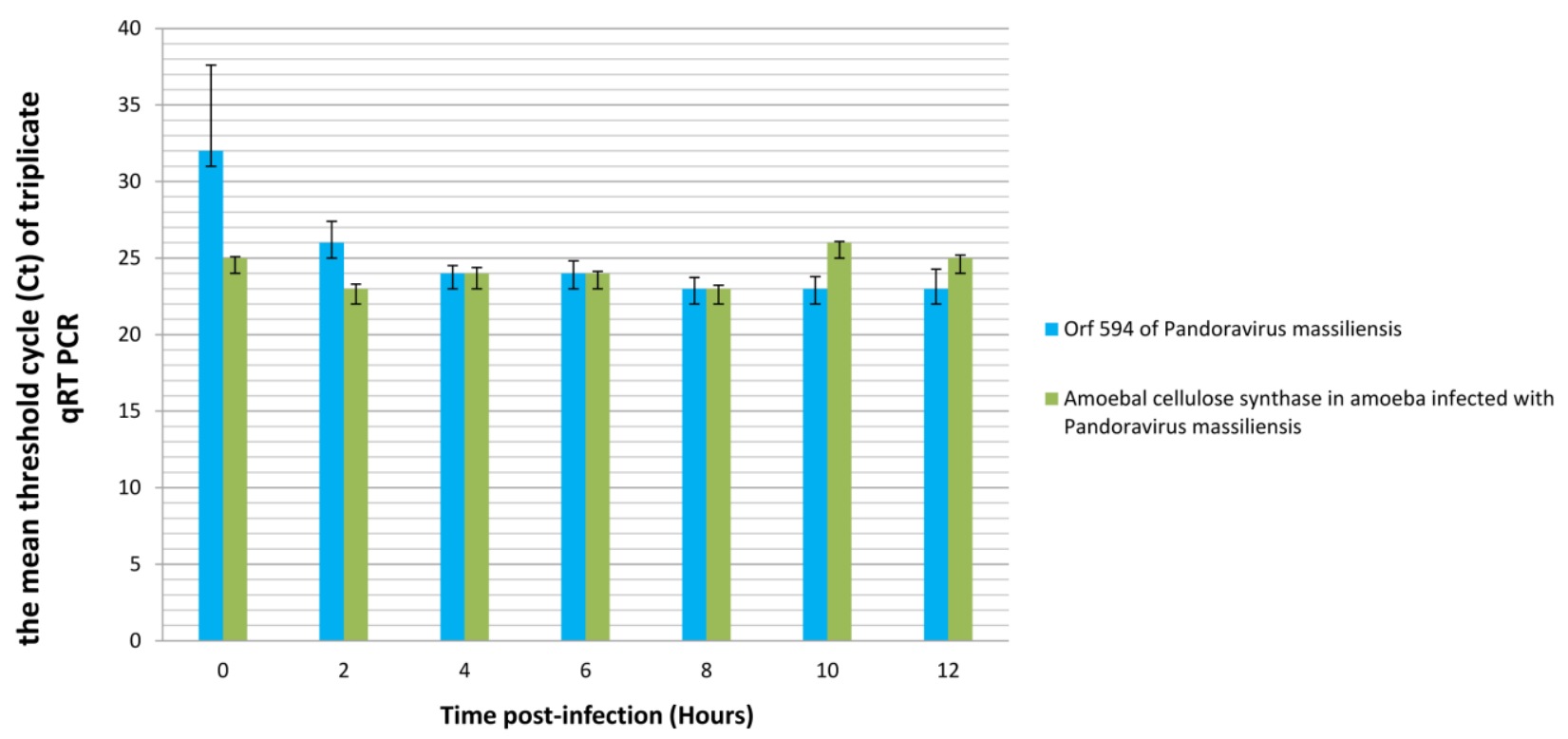

496 Figure 11. Representation of the mean threshold cycle $(\mathrm{Ct})$ of triplicate qRT PCR on the RNA of

497 Pandoravirus massiliensis by targeting the predicted gene of the cellulose synthase (ORF594) and on

498 RNA of Acanthamoeba castellanii infected with Pandoravirus massiliensis by targeting the amoebal

499 gene of the cellulose synthase, according to the time post-infection from 0 to 12 hours post-

500 infection). 